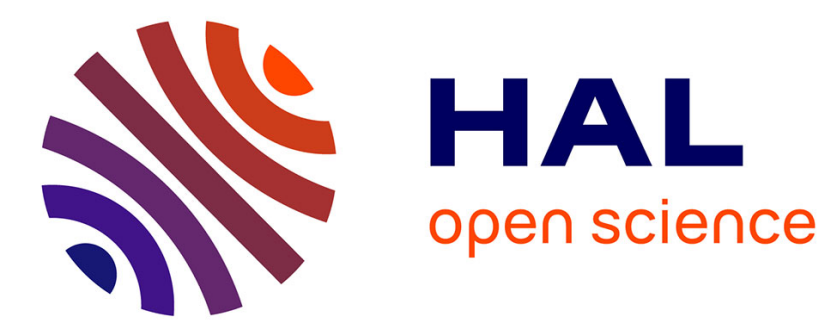

\title{
Nanostructured diode for infrared photodetection through nondegenerate two-photon absorption
}

Baptiste Fix, Julien Jaeck, Benjamin Vest, Michael Verdun, Grégoire Beaudoin, Isabelle Sagnes, Jean-Luc Pelouard, Riad Haïdar

\section{To cite this version:}

Baptiste Fix, Julien Jaeck, Benjamin Vest, Michael Verdun, Grégoire Beaudoin, et al.. Nanostructured diode for infrared photodetection through nondegenerate two-photon absorption. Applied Physics Letters, 2017, 111, pp.041102-1 - 041102-5. 10.1063/1.4996369 . hal-01709018

\section{HAL Id: hal-01709018 https://hal.science/hal-01709018}

Submitted on 22 Nov 2021

HAL is a multi-disciplinary open access archive for the deposit and dissemination of scientific research documents, whether they are published or not. The documents may come from teaching and research institutions in France or abroad, or from public or private research centers.
L'archive ouverte pluridisciplinaire HAL, est destinée au dépôt et à la diffusion de documents scientifiques de niveau recherche, publiés ou non, émanant des établissements d'enseignement et de recherche français ou étrangers, des laboratoires publics ou privés.

\section{(ㅇ)(1) $\$$}

Distributed under a Creative Commons Attribution - NonCommercial| 4.0 International 


\title{
Nanostructured diode for infrared photodetection through nondegenerate two-photon absorption
}

\author{
Baptiste Fix, ${ }^{1}$ Julien Jaeck, ${ }^{1, a)}$ Benjamin Vest, ${ }^{1, b)}$ Michaël Verdun, ${ }^{2}$ Grégoire Beaudoin, ${ }^{3}$ \\ Isabelle Sagnes, ${ }^{3}$ Jean-Luc Pelouard, ${ }^{2}$ and Riad Haïdar ${ }^{1, c)}$ \\ ${ }^{1}$ ONERA-The French Aerospace Lab, Chemin de la Hunière, 91761 Palaiseau Cedex, France \\ ${ }^{2} \mathrm{MiNaO}$-Center for Nanoscience and Nanotechnology (C2N)-CNRS, Univ. Paris-Sud, \\ Université Paris-Saclay, 91460 Marcoussis, France \\ ${ }^{3}$ Center for Nanoscience and Nanotechnology (C2N)-CNRS, Univ. Paris-Sud, \\ Université Paris-Saclay, 91460 Marcoussis, France
}

(Received 5 May 2017; accepted 16 July 2017; published online 25 July 2017)

\begin{abstract}
We investigate infrared detection at room temperature using non-degenerate two-photon absorption in a nanostructured indium phosphide photodiode. We designed the detector structure to achieve a good nonlinear absorption by combining three major ideas: first, we use the non-degenerate twophoton absorption process, which is known to be more efficient than the previously used degenerate two-photon absorption. Second, we ensured a correct spatial overlap of our pump field with our signal field. Third, we optimized the nanostructuration to increase the signal field amplitude locally within the active medium of the device. The resulting device consists of a PIN junction embedded between a back-reflecting gold mirror and a top grating. We experimentally characterized our diode with regard to reflectivity and two-photon absorption generated photocurrent for a continuouswave pump and a nanosecond pulsed signal of around $3.39 \mu \mathrm{m}$. Owing to the nanostructuration, the generated photocurrent shows a gain of 24 with respect to the bulk response of InP. Published by AIP Publishing. [http://dx.doi.org/10.1063/1.4996369]
\end{abstract}

Two-photon absorption (TPA) is a third order nonlinear process that relies on the quasi-simultaneous absorption of two photons. Therefore, it has proven to be an interesting tool to measure ultra-fast correlations ${ }^{1}$ or to design alloptical switches; ${ }^{2}$ yet, due to the intrinsically low efficiency of the nonlinear processes, these applications rely upon highpeak power light sources, such as femtosecond and picosecond pulsed lasers. However, TPA has also been noticed as an appealing alternate scheme for quantum infrared detection. ${ }^{3,4}$ Indeed, typical quantum detection of IR radiation is based on small gap semiconductors that need to be cooled down to a cryogenic temperature to achieve sufficient detectivity. TPA enables the absorption of IR photons by wide gap semiconductors when pump photons are provided to complete optical transitions across the gap. Still, the low efficiency of TPA represents a difficulty to detect usual infrared photon fluxes. To tackle this issue, we combined three strategies to improve the detection efficiency. First, it has been proven theoretically and experimentally that using different pumps and signal photon energies, which is known as non-degenerate TPA (NDTPA), helps to increase the TPA efficiency by several orders of magnitude. ${ }^{5}$ Thus, we decided to work with different pumps and signal wavelengths. Second, since TPA is a local quasi-instantaneous process, both pump and signal photons must be temporarily and spatially co-localized within the active medium. We made sure that the overlap of the fields inside our device was maximized. Finally, it is well

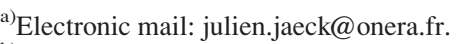

b) Present address: Laboratoire Charles Fabry, 2 avenue Augustin Fresnel, 91127 Palaiseau Cedex, France.

c) Present address: Département de Physique, École Polytechnique, Université Paris-Saclay, 91128 Palaiseau, France.
}

known that TPA has a quadratic dependence with the signal electric field modulus, so we designed a specific nanostructure to enhance the signal field within the active medium of the detector.

In this letter, we report the experimental demonstration of enhanced non-degenerate two-photon absorption in an indium phosphide (InP) PIN diode. We have nanostructured the front electrode, so that the pump and the signal waves are confined and overlap within the intrinsic zone of the diode. We have experimentally characterized the behavior of the photocurrent generation with respect to the signal wavelength and polarization. Moreover, we have checked the linearity of the photocurrent with respect to the pump and the signal intensities. Confinement within the nanostructure leads to a 24 time increase of the two-photon current generation, as compared to the bulk, which is close to the simulation results.

We have numerically designed the structure using the linear B-Spline modal method. ${ }^{3,6}$ The periodic 1D structure parameters, detailed in Fig. 1 , are $h_{\mathrm{Au}}=100 \mathrm{~nm}, \mathrm{~L}=700 \mathrm{~nm}$, $\mathrm{p}=1780 \mathrm{~nm}$, and $\mathrm{w}=580 \mathrm{~nm}$. The nominal thicknesses of the $\mathrm{p}$-i-n junction are $50 \mathrm{~nm}$ for the p-layer, $480 \mathrm{~nm}$ for the ilayer, and $50 \mathrm{~nm}$ for the $\mathrm{n}$-layer. The $\mathrm{n}(\mathrm{p})$-layer is doped with a density of $1 \times 10^{18} \mathrm{~cm}^{-3}$, while the i-layer has an n-type residual doping with a density of about $1 \times 10^{16} \mathrm{~cm}^{-3}$. For a TE polarized wave under normal incidence, this structure exhibits a guided mode resonance $e^{7,8}$ at the signal wavelength $(\lambda=3.39 \mu \mathrm{m})$, resulting in a critical coupling between free space and the device. As it is shown in Fig. 1, the computed reflectivity of this resonance is characterized by a decrease in the reflectivity due to the dissipation in the metal; moreover, it exhibits a convenient angular tolerance (up to $\pm 10^{\circ}$ of incidence). Under $11^{\circ}$ incidence, the TE-polarized pump light at 

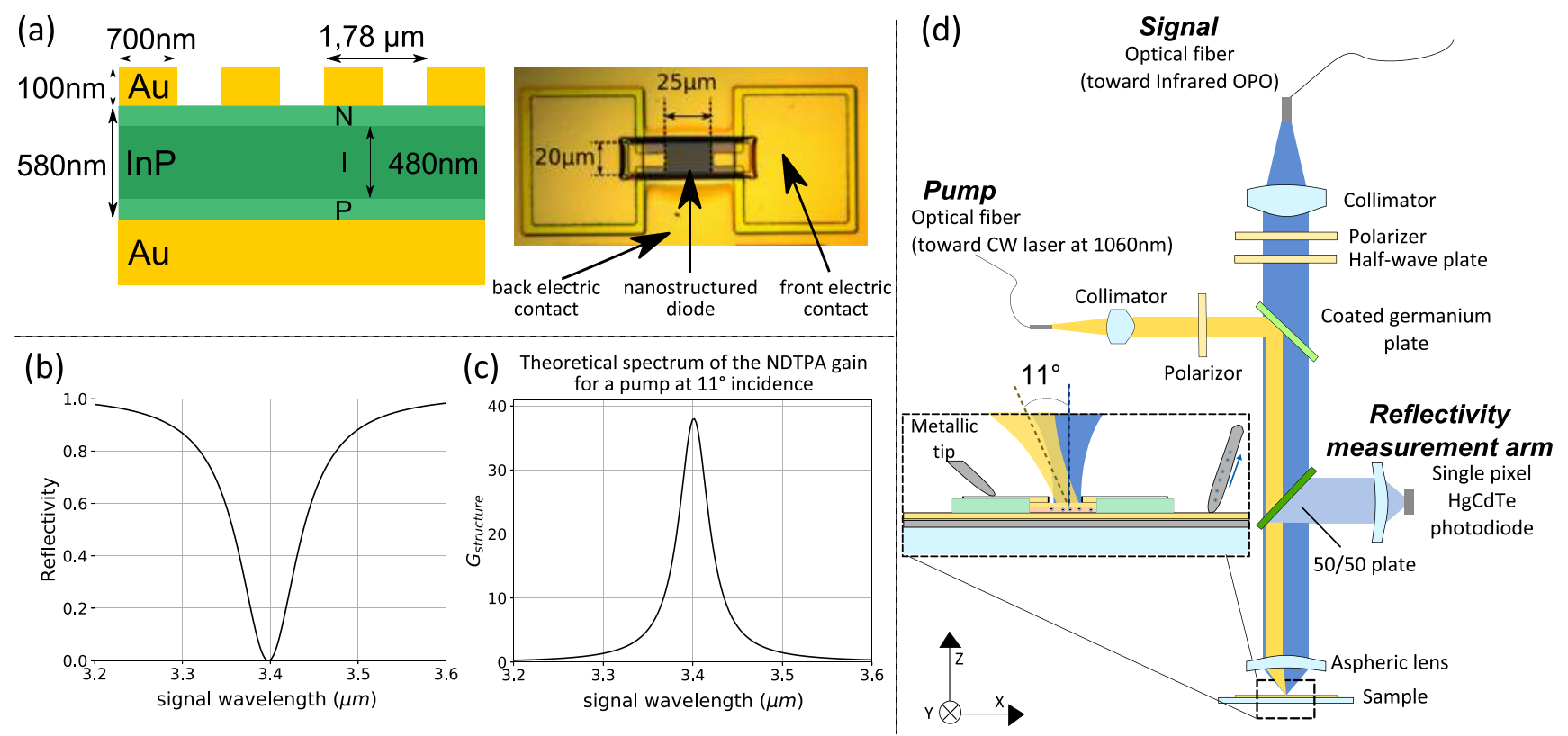

(b)

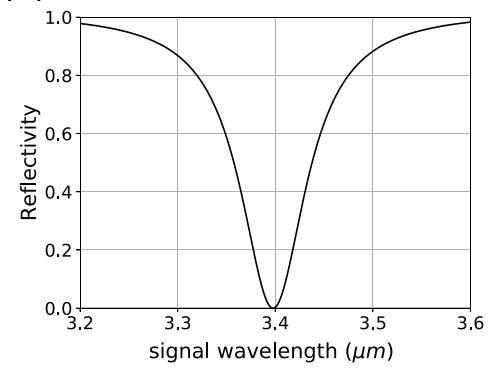

(c) Theoretical spectrum of the NDTPA gain

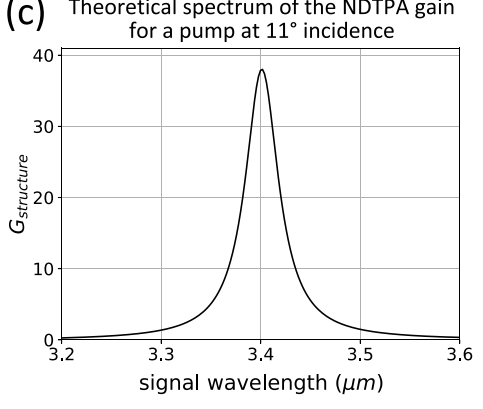

(d)

FIG. 1. (a) On the left is a cross sectional schematic of the diode. An indium phosphide PIN diode of 480 nm non-intentionally doped zone is placed between a back mirror and a front nanostructured electrode. On the right is an annotated picture taken with a microscope from one realization of the diode. In the center is the nanostructured electrode of dimensions $20 \times 25 \mu \mathrm{m}$. On both sides are gold pads to place the metallic tip on and to achieve one of the electric contacts. (b) Theoretical reflectivity of the structure for a TE polarized signal light. (c) Evolution of the expected gain on the two-photon current $G_{\text {structure with the signal }}$ wavelength for a pump at $11^{\circ}$ incidence. (d) Schematic diagram of the dedicated bench. The sample is fixed to the table and the whole optical cage can be translated along the three axes $\mathrm{X}, \mathrm{Y}$, and $\mathrm{Z}$.

$1.06 \mu \mathrm{m}$ is coupled to a delocalized mode guided inside the diode, which has a relatively homogeneous field distribution inside the diode.

In order to optimize this nanostructure, we relied upon the NDTPA theory. This nonlinear absorption is locally proportional to the product of the pump and signal field intensities. Thus, in every position of the intrinsic zone of the diode, the local charge generation $j_{\mathrm{NDTPA}}$ is driven by the equation $j_{\mathrm{NDTPA}}=\beta I_{p} I_{s}$, where $I_{p}\left[\mathrm{~W} / \mathrm{m}^{2}\right]\left(\right.$ resp. $\left.I_{s}\right)$ is the local pump (resp. signal) intensity and $\beta$ is the two photon absorption coefficient. The nanostructure usefulness is therefore to locally concentrate the signal field within the i-zone of the diode. Taking into account the macroscopic charge collection, we can write the integrated NDTPA photocurrent generation $J_{\mathrm{NDTPA}}$ as $J_{\mathrm{NDTPA}}=\beta G_{\text {structure }} I_{p}^{(i)} I_{s}^{(i)}$, where $I_{p}^{(i)}\left(\right.$ resp. $\left.I_{s}^{(i)}\right)$ are the incident pump (resp. signal) intensities, and $G_{\text {structure }}$ is the gain due to the nanostructuration defined as

$$
G_{\text {structure }}=\frac{\left\langle I_{p} I_{s}\right\rangle}{I_{p}^{(i)} I_{s}^{(i)}},
$$

where $\langle\cdot\rangle$ represent the spatial integration in the i-layer over a period of the nanostructure. This theoretical gain is easily computed in the BMM B-spline modal method (BMM) method and was used as a figure of merit for the design optimization. The computed spectral evolution of this gain for the final structure is illustrated in Fig. 1(c). It reaches a maximum of 38 at $3.39 \mu \mathrm{m}$, meaning that we expect the photocurrent generation to be 38 times higher due to the nanostructure.

The experimental setup, presented in Fig. 1(d), consists of three elements: the signal source, the pump source, and the reflectivity measurement arm. The signal source is an MSquared Firefly-IR optical parametric oscillator (OPO), delivering pulses with a duration of $\Delta \mathrm{t}=10 \mathrm{~ns}$ with a repetition rate of $f=150 \mathrm{kHz}$. It is coupled to a fiber, and its linear polarization is controlled through a combination of a polarizer and a half-wave plate. An aspheric lens (focal length: $6 \mathrm{~mm}$ ) is used to focus the light into a spot of diameter $25 \mu \mathrm{m}$ (measured at $\frac{1}{e^{2}}$ by a knife-edge method) on the photodiode. The pump light is emitted by a fibered continuous wave laser diode (Thorlabs LPS-1060-FC) that is also linearly polarized. The pump beam is decentered from the focusing lens in order to achieve an $11^{\circ} \pm 2^{\circ}$ incidence, as needed to achieve the overlap. The whole optical cage is mounted on a motorized three axis translation stage on top of a CascadeMicrotech EP9 probe station. The diode is connected by metallic probes to a subfemtoamp sourcemeter Keithley 6430, which applies a bias voltage of $-100 \mathrm{mV}$ and collects the generated photocurrent. All of the photocurrent measurements were performed at room temperature. The infrared reflectivity measurements were performed with a thermoelectrically cooled $\mathrm{HgCdTe}$ single-pixel photodetector.

We designed the confining nanostructure to have a specific linear response of the InP device at the signal wavelength $(3.39 \mu \mathrm{m})$. To check the optical behavior of our device, we first measure the reflectivity of the device in the vicinity of the signal wavelength without the pump source. We perform a reflectivity scan of the device by translating the optical cage in the $(\mathrm{X}, \mathrm{Y})$ plane with motorized stages. Figure 2(a) insets show the reconstructed images in reflection at the signal wavelength. Each $(i, j)$ pixel shows the mean intensity for a light spot centered at $\left(X_{i}, Y_{i}\right)$. In these pictures, one can easily identify the sample, the two lateral pads, and one of the probes thanks to the reflection on the sloping edges. We obtain the diode reflectivity value by assuming that, far from the structure, the back gold mirror can be considered as a reflectivity reference. By comparing the images of a nanostructured diode and a naked one, we 
(a)

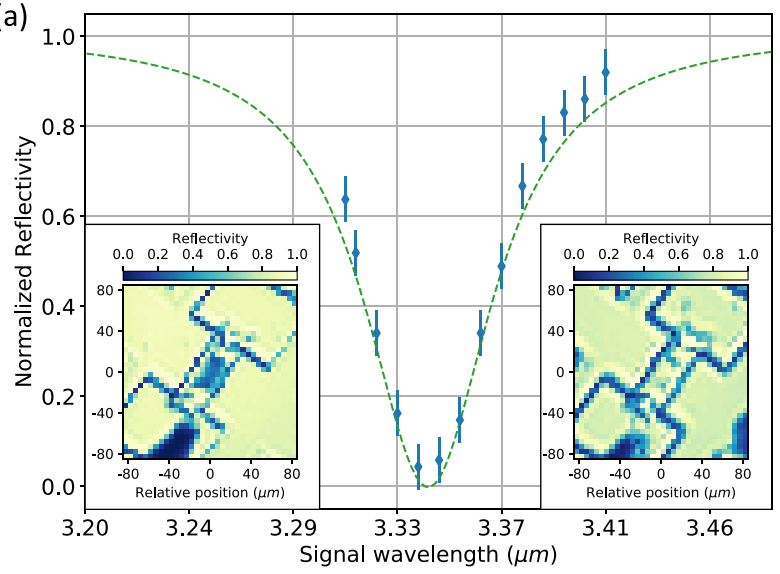

(b)

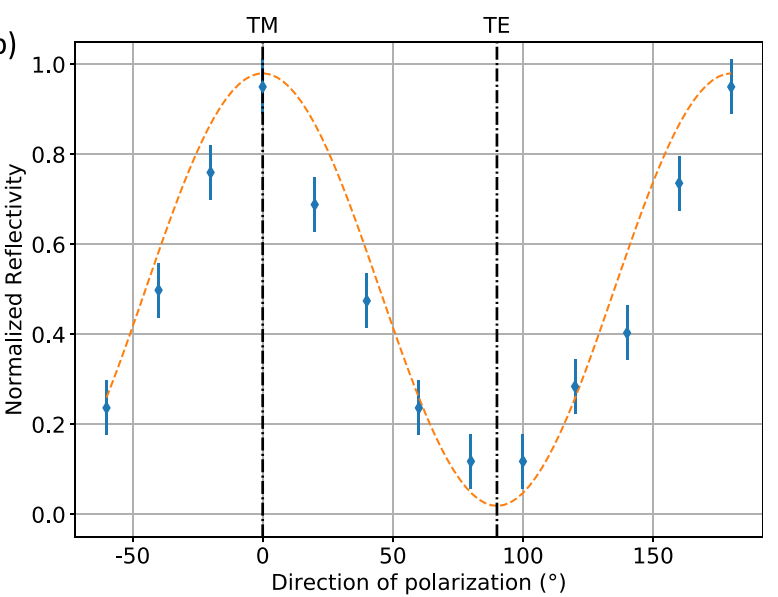

FIG. 2. (a) Spectral characterization of the diode reflectivity for a TE polarized signal beam at normal incidence. The dotted line is the computed reflectivity, as obtained by modal calculations. The diamonds are experimental data. The resonance is shifted to $3.36 \mu \mathrm{m}$ as compared with the theory, due to the manufacturing imprecision. The insets represent the reconstructed reflectivity images of two diodes at $3.36 \mu \mathrm{m}$. On the left, the diode is nanostructured, and the scan shows a clear decrease in the reflectivity at the position of the structure. On the right is a naked diode (i.e., without the top grating) showing no decrease in the reflectivity. (b) Characterization of the reflectivity with respect to the incident direction of polarization at $3.36 \mu \mathrm{m}$. The diamonds are experimental data, and the dotted line is a sinusoidal eye-guide. As expected, the reflectivity of the diode is at a minimum for a TE-polarized light and at a maximum for a TMpolarized light.

see a decrease in the reflectivity due to the electrode structuration. Using the OPO signal source, we measure the spectral evolution of the structured diode reflectivity, as shown in Fig. 2(a). One can note that the resonating wavelength is actually at $3.36 \mu \mathrm{m}$, which represents a small spectral shift compared to the theoretical value given previously. We attribute this to the manufacturing imprecision. Indeed, this shift corresponds to an effective width of $750 \mathrm{~nm}$ for the nanobars. We use this new value from now on. Figure 2(b) shows the dependence of the reflectivity with the direction of polarization at $3.36 \mu \mathrm{m}$. As expected, the reflectivity of the diode is at a minimum for a TE-polarized light and at a maximum for a TM-polarized light.

Having characterized the linear behavior of the cavity at the signal wavelength, we can now study the photocurrent generation through NDTPA. This nonlinear process is subjected to the simultaneous presence and co-localization of signal and pump sub-bandgap photons inside the intrinsic semiconductor. With both sources turned on, the expected photocurrent generation is a sum of three terms: (1) the linear absorption current, (2) the degenerate TPA current of the pump, and (3) the NDTPA-generated photocurrent

$$
J_{\text {total }}=\alpha\left(\omega_{p}\right)\left\langle I_{p}\right\rangle+\beta\left(\omega_{p}, \omega_{p}\right)\left\langle I_{p}^{2}\right\rangle+\beta\left(\omega_{p}, \omega_{s}\right)\left\langle I_{p} I_{s}\right\rangle,
$$

where $I_{p}$ (resp. $I_{s}$ ) is the intensity $\left[\mathrm{W} / \mathrm{m}^{2}\right]$ of the pump (resp. signal) beam, $\langle\cdot\rangle$ represents the integration in the i-layer, $\alpha$ is the linear absorption coefficient, and $\beta$ is the TPA coefficient. The first term of the photocurrent comes from a linear absorption of sub-bandgap photons from the pump light. This phenomenon has been studied in several materials (silicium and gallium arsenide) and is explained as a photo-assisted Shockley-Read-Hall process. ${ }^{9,10}$ The second term is the degenerate two-photon absorption of the pump. ${ }^{3}$ Since these two contributions are related to the absorption of the pump only, using the usual hypothesis of an undepleted pump, we perform a calibration step by measuring the current inside the junction without any signal photons. The NDTPA-generated photocurrent is then obtained from the difference between the generated photocurrent due to the pump light alone and that generated under both the pump and signal illumination $J_{\text {NDTPA }}$ $=J_{\text {total }}-\alpha\left(\omega_{p}\right)\left\langle I_{p}\right\rangle-\beta\left(\omega_{p}, \omega_{p}\right)\left\langle I_{p}^{2}\right\rangle$. We show in Fig. 3 the spectrum of the NDTPA-generated photocurrent and its dependence with the direction of polarization of the signal at $3.36 \mu \mathrm{m}$, which shows a maximum efficiency for the TE polarized signal. For this configuration, we measured a signalto-noise ratio higher than 15 for mean incident powers of 1.4 $\mathrm{mW}$ for the signal and $1.3 \mathrm{~mW}$ for the pump [see Fig. 3(c)]. In the same configuration, no signal above the noise was measurable for a diode without the top grating. As expected, the spectrum of the NDTPA-generated photocurrent follows that of the nanostructure except for the re-bounce at $3.4 \mu \mathrm{m}$ that we partially attribute to the numerical aperture of 0.53 of the focused beam and the angular response of the nanostructure. Finally, we characterized the NDTPA-generated photocurrent evolution with either the pump or the signal incident power for a TE-polarized signal at $3.36 \mu \mathrm{m}$. The plots in Fig. 4 show the linear behavior of the generated photocurrent with respect to the pump and signal intensities confirming its NDTPA nature. From these data, we derived a linear fitting parameter of $77 \pm 5 \mu \mathrm{A} / \mathrm{W}^{2}$. Theoretically, the NDTPA-generated photocurrent inside the structure can be expressed as ${ }^{3}$

$$
J_{\mathrm{NDTPA}}=\beta\left(\omega_{p}, \omega_{s}\right) \frac{\eta e}{\hbar \omega_{s}} \frac{G_{\text {structure }} h_{\text {i-layer }}}{S_{\text {diode }}} P_{p} P_{s},
$$

where $\eta$ is the charge extraction efficiency, $S_{\text {diode }}=500$ $\times 10^{-6} \mathrm{~mm}^{2}$ is the surface of the diode, $h_{\mathrm{i} \text {-layer }}=480 \mathrm{~nm}$ is the height of the intrinsic zone of the diode, and $P_{p}$ (resp. $P_{s}$ ) is the mean power of the pump (resp. signal) beam. $G_{\text {structure }}$ is the NDTPA gain coefficient due to the cavity. We can thus obtain $\beta_{\text {eff }}=G_{\text {structure }} \beta\left(\omega_{p}, \omega_{s}\right)=2950 \pm 200 \mathrm{~cm} / \mathrm{GW}$. We were unable to find any similar non-degenerate coefficient in the literature to compare with our measurements. However, using the validated TPA coefficient theory ${ }^{11}$ [see Eq. (4)] and the latest values of TPA for InP, ${ }^{12,13}$ we have derived an approximate NDTPA coefficient 
(a)

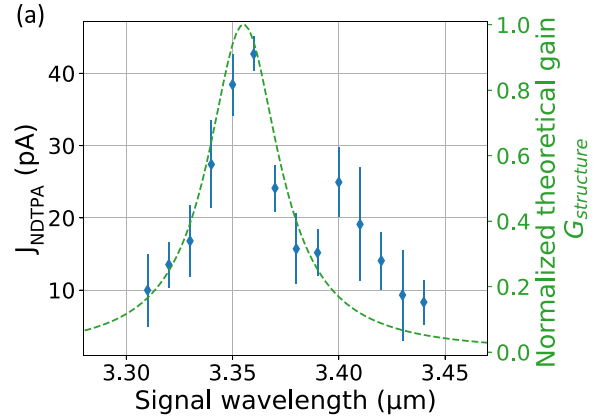

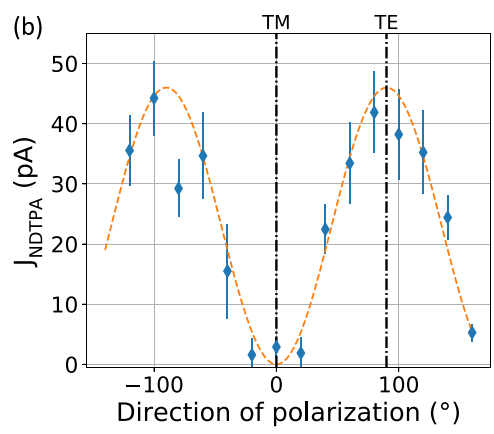

FIG. 3. (a) Spectral evolution of the NDTPA-generated photocurrent for a mean incident power of $300 \mu \mathrm{W}$ for the pump and $1.4 \mathrm{~mW}$ for the signal. The measurements follow the theoretical linear gain of the nanostructure. We attribute the re-bounce to the signal-beam angular spectrum. (b) Dependence of the NDTPA-generated photocurrent with the signal direction of polarization for the same experimental parameters and a signal at $3.36 \mu \mathrm{m}$. The maximum of NDTPA-generated photocurrent is achieved for a TE polarized signal. The dotted line is a sinusoidal eye-guide. (c) Scanned map of the signal-to-noise ratio measured for a TE polarized signal at $3.36 \mu \mathrm{m}$. The incident signal mean power is $1.4 \mathrm{~mW}$ and the incident pump mean power is $1.3 \mathrm{~mW}$. The white line is the diode skeleton as measured simultaneously through reflection, which has been added as a visual guide.
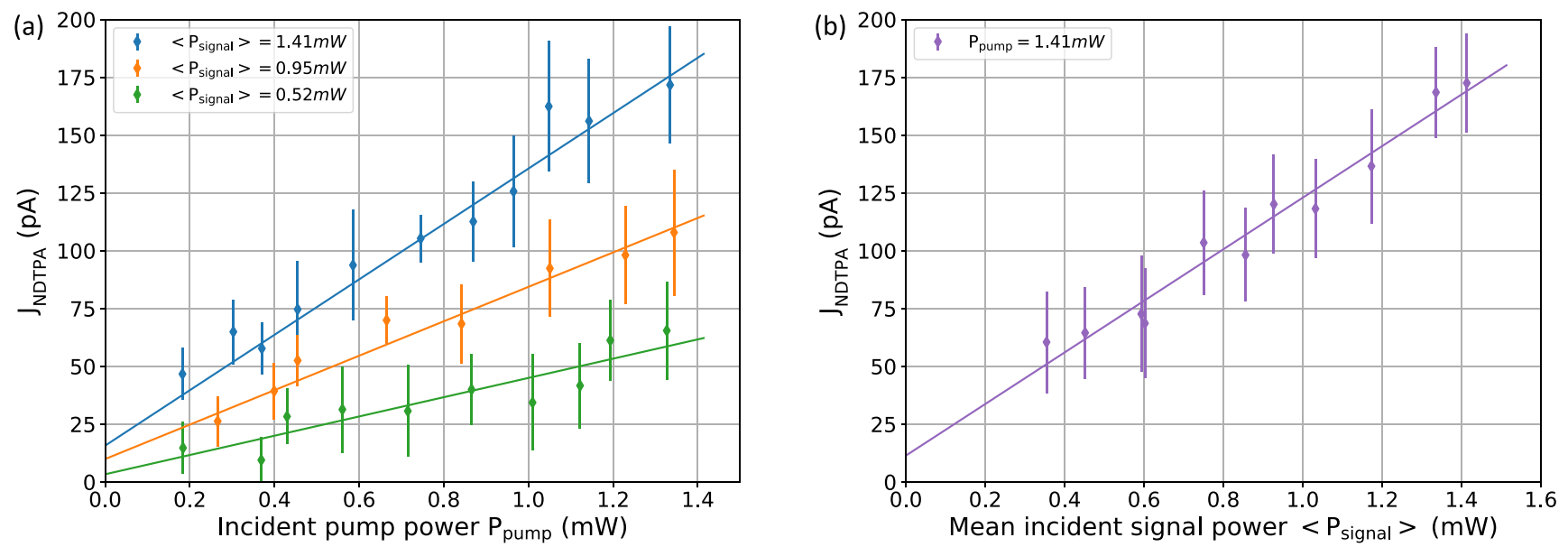

FIG. 4. Characteristics of the generated photocurrent for a TE-polarized signal at $3.36 \mu \mathrm{m}$ with (a) the incident pump power and with (b) the incident signal power. The lines are the least squares regression from the experimental data points, and the error-bars represent the temporal measurement noise.

$$
\begin{gathered}
\beta\left(\omega_{p}, \omega_{s}\right)=\frac{Q \sqrt{E_{p}}}{n_{p} n_{s} E_{g}^{3}} F_{2}\left(\frac{\hbar \omega_{p}}{E_{g}}, \frac{\hbar \omega_{s}}{E_{g}}\right) \\
\text { with } F_{2}\left(x_{1}, x_{2}\right)=\frac{\left(x_{1}+x_{2}-1\right)^{\frac{3}{2}}}{2^{7} x_{1} x_{2}^{2}}\left(\frac{1}{x_{1}}+\frac{1}{x_{2}}\right)^{2},
\end{gathered}
$$

where $\mathrm{Q}$ is a material-independent constant of typical value $3100 \frac{\mathrm{cmeV}^{5 / 2}}{\mathrm{GW}}, E_{p}=21 \mathrm{eV}$ is almost material independent, $E_{g}=1.344 \mathrm{eV}$ is the bandgap, and $n_{p}$ and $n_{s}$ are the refractive indices of InP at $\omega_{p}$ and $\omega_{s}$, respectively. We obtain a value of $\beta(1.06 \mu \mathrm{m}, 3.36 \mu \mathrm{m}) \simeq 122 \mathrm{~cm} / \mathrm{GW}$. If we compare the value of $\beta$ with the effective coefficient that we measured in our sample, we can calculate the NDTPA gain due to the nanostructure: $G_{\text {structure }}=\frac{\beta_{\text {eff }}}{\beta}=24 \pm 1.6$.

In summary, a nanostructured infrared photodetector through non-degenerated two-photon absorption has been designed, manufactured, and characterized. We demonstrated the detection of a $3.39 \mu \mathrm{m}$ nanosecond pulsed signal at room temperature inside a thin indium phosphide PIN junction with a signal-to-noise ratio of 15 . We proved that the electrode nanostructuration creates a gain on the NDTPA efficiency of 24. By enabling a clear increase in the NDTPA responsivity, the nanostructuration of wide-gap semiconductor based photodiodes paves the way toward continuous wave infrared detection at room temperature. To further improve the device responsivity, we can imagine a biresonant nanostructure at the signal wavelength and at the pump wavelength which would improve the overlap of the two fields and would decrease the PASRH photocurrent.

This research was supported by a DGA-MRIS scholarship and was partially supported by the French RENATECH network.

${ }^{1}$ F. Boitier, A. Godard, E. Rosencher, and C. Fabre, Nat. Phys. 5, 267 (2009). ${ }^{2}$ S. M. Hendrickson, C. N. Weiler, R. M. Camacho, P. T. Rakich, A. I. Young, M. J. Shaw, T. B. Pittman, J. D. Franson, and B. C. Jacobs, Phys. Rev. A 87, 023808 (2013).

${ }^{3}$ B. Portier, B. Vest, F. Pardo, N. Péré-Laperne, E. Steveler, J. Jaeck, C. Dupuis, N. Bardou, A. Lemaître, E. Rosencher, R. Haïdar, and J.-L. Pelouard, Appl. Phys. Lett. 105, 011108 (2014).

${ }^{4}$ D. Fishman, C. M. Cirloganu, S. Websterand, L. A. Padilha, M. Monroe, D. J. Hagan, and E. W. V. Stryland, Nat. Photonics 5, 561 (2011).

${ }^{5}$ C. M. Cirloganu, L. A. Padilha, D. A. Fishman, S. Webster, D. J. Hagan, and E. W. V. Stryland, Opt. Express 19, 22951 (2011).

${ }^{6}$ P. Bouchon, F. Pardo, R. Haïdar, and J.-L. Pelouard, J. Opt. Soc. Am. A 27, 696 (2010).

${ }^{7}$ R. Magnusson and S. S. Wang, Appl. Phys. Lett. 61, 1022 (1992). 
${ }^{8}$ E. Sakat, G. Vincent, P. Ghenuche, N. Bardou, S. Collin, F. Pardo, J.-L. Pelouard, and R. Haïdar, Opt. Lett. 36, 3054 (2011).

${ }^{9}$ B. Vest, E. Lucas, J. Jaeck, R. Haïdar, and E. Rosencher, Appl. Phys. Lett. 102, 031105 (2013).

${ }^{10}$ B. Vest, B. Fix, J. Jaeck, and R. Haïdar, J. Eur. Opt. Soc.-Rapid Publ. 12, 26 (2016).
${ }^{11}$ E. W. V. Stryland, M. A. Woodall, H. Vanherzeele, and M. J. Soileau, Opt. Lett. 10, 490 (1985).

${ }^{12}$ T. Matsusue, H. Bando, S. Fujita, and Y. Takayama, Phys. Status Solidi C 8, 387 (2011).

${ }^{13}$ D. Vignaud, J. F. Lampin, and F. Mollot, Appl. Phys. Lett. 85, 239 (2004). 